\title{
Evaluación de resinas de intercambio iónico para el control del hierro en soluciones de electro-obtención de cobre ${ }^{(\bullet)}$
}

\author{
F. Parada*, D. Dreisinger** e I. Wilkomirsky*
}

\begin{abstract}
Resumen
Se evaluaron dos resinas comerciales para la extracción del hierro desde una solución de electro-obtención de cobre. Ambas resinas extraen en forma eficiente el hierro, pero se diferencian en que la resina Monophosphonic tiene una mayor capacidad de carga que la resina Diphonix, mientras que la Diphonix muestra una cinética más rápida. Los resultados de la prueba interrumpida y de las pruebas para determinar el efecto del tamaño de partícula permiten concluir que el control más probable para la cinética de extracción es por la difusión en la partícula en ambas resinas. Un buen ajuste al modelo de Fick para difusión en la partícula confirman el tipo de control propuesto. Finalmente, la temperatura favorece la cinética del proceso de extracción y su efecto en el coeficiente de difusión sigue la ley de Arrhenius obteniendo un valor para la energía de activación de 4,89 kcal/mol para la resina Monophosphonic y 4,94 $\mathrm{kcal} / \mathrm{mol}$ para la resina Diphonix. Estos valores están cercanos al valor típico para el control propuesto que es de 6 a $10 \mathrm{kcal} / \mathrm{mol}$
\end{abstract}

\section{Evaluation of ion exchange resins for iron control in copper electro-winning solutions}

\begin{abstract}
Two commercial resins were evaluated for the extraction of iron from a copper electrowinning solution. Both resins efficiently extract iron. The Monophosphonic resin has a greater charge capacity than the Diphonix resin and the Diphonix resin shows faster kinetics. Experimental results of the interrupted test and tests with different particle size of resins have demonstrated that extraction kinetics is controlled by diffusion into the particle in both resins. A good agreement with Fick's model for diffusion inside the particles confirms the proposed mechanism. Finally, temperature favors the process kinetics and its effect on the diffusion coefficient follows Arrhenius law, obtaining a value of 4,89 kcal/mol for the Monophosphonic resin and 4,94 kcal/mol for the Dphonix resin. The aforementioned values are close to typical values for the proposed diffusional control which are 6 to $10 \mathrm{kcal} / \mathrm{mol}$.
\end{abstract}

Keywords

Ion exchange; Iron control; Copper electrowinning.

\section{INTRODUCCIÓN}

La eliminación de hierro desde soluciones ácidas utilizando resinas de intercambio iónico se ha probado con éxito desde comienzo de los 90 ${ }^{[1]}$. La primera aplicación comercial de esta tecnología fue en la planta de extracción por solvente-electro-obtención de Cananea en México, en que se utilizó una resina comercial (Diphonix). Esta resina contiene grupos de ácido fosfónico que son los que le dan la capacidad de extraer Fe(III) en forma selectiva con respecto a iones di y monovalentes ${ }^{[2]}$. Además de esta resina, se desarrolló una resina (Monophosphonic) que posee los mismos grupos funcionales que la resina Diphonix, pero con un mayor contenido de ácido fosfónico, la cual se está utilizando comercialmente para purificar el electrolito de electro-obtención de cobre en la planta Mt. Gordon de Western Metals Copper Ltd en Queensland Australia ${ }^{[3]}$.

La aplicación de esta tecnología en una planta permite minimizar los descartes de electrolito que se realizan en plantas de extracción por solvente-electro-obtención (SX - EW) para evitar que aumente el contenido de impurezas. Estos descartes son el

\footnotetext{
(•) Trabajo recibido el día 13 de Agosto de 2009 y aceptado en su forma final el día 16 de Abril de 2010.

* Departamento de Ingeniería Metalúrgica, Universidad de Concepción, Chile.

${ }^{* *}$ Metals and Materials Engineering Department, University of British Columbia, Canada.
} 
principal motivo de las pérdidas de reactivos utilizados en el proceso de EW de cobre, dentro de los cuales el más importante es el sulfato de cobalto.

Otra aplicación que, además, es el origen de este estudio, es en un nuevo proceso desarrollado en la Universidad de Concepción (Chile) para tratar eje o metal blanco de cobre mediante un proceso de Tostación - Lixiviación - EW [4]. Este proceso genera soluciones concentradas en cobre y con muy bajos niveles de impurezas que pueden enviarse directamente a electro-obtención evitando la etapa de SX. En estas condiciones, todo el electrolito se recircula a lixiviación y, por tanto, cualquier descarte de electrolito para mantener el control del hierro en la solución tendría que procesarse aparte, para evitar pérdidas de cobre y ácido contenidos en el electrolito. Para esta aplicación, la eliminación de hierro con resinas es la mejor opción tecnológica para controlar el nivel de hierro y, así, poder retornar el descarte de electrolito a la etapa de lixiviación.

Se planteó, así, un estudio de evaluación de dos resinas (Monophosphonic y Diphonix) para el control del hierro en soluciones ácidas obtenidas en la lixiviación de calcinas oxidadas de eje de cobre.

\section{CARACTERIZACION DE LAS RESINAS}

En la tabla I se presenta la composición química de las dos resinas de intercambio iónico utilizadas en este estudio. Se puede observar que la resina Monophosphonic contiene más del doble de contenido de fósforo que la Diphonix, lo que significa un mayor contenido de los grupos de ácido fosfónico que son selectivos por el Fe(III), mientras que la resina Diphonix muestra un mayor contenido de azufre, producto de un mayor contenido de grupos de ácido sulfónico, el cual acelera la cinética del proceso ${ }^{[2 \text { y } 5]}$.

Las concentraciones de los elementos adsorbidos en las resinas se miden con respecto a la masa de resina seca a $65^{\circ} \mathrm{C}$ y su equivalencia con respecto al volumen de resina en medio acuoso (lecho de resina), como se muestra en la tabla II.

\section{METODOLOGIA EXPERIMENTAL}

\subsection{Pruebas de equilibrio}

Se realizaron en un agitador de movimiento orbital (shaker) con velocidad variable hasta $300 \mathrm{rpm}$ y control de temperatura que permitía llegar hasta los $90^{\circ} \mathrm{C}$ y que podía operar simultáneamente con nueve matraces erlenmeyer de $250 \mathrm{~cm}^{3}$. La solución acuosa se preparó de acuerdo a un electrolito agotado de electro-obtención con $35 \mathrm{~g} / \mathrm{l}$ de cobre y $180 \mathrm{~g} / \mathrm{l}$ de ácido sulfúrico, agregando cantidades variables de sulfato de hierro (III) para obtener concentraciones deseadas de férrico. Las variables consideradas y sus valores son:

Resina Monophosphonic:

$\begin{array}{ll}\mathrm{Fe}^{3+}(\mathrm{ppm}) & : 1.000,1.500 \text { y } 2.000 \\ \text { Temperatura }\left({ }^{\circ} \mathrm{C}\right) & : 20 ; 35 ; 45 ; 55 \\ \text { Razón acuoso/resina (vol.) } & : 10 / 1 \text { a } 500 / 1 \\ \text { Tiempo (h) } & : 24\end{array}$

Resina Diphonix:

$\begin{array}{ll}\mathrm{Fe}^{3+}(\mathrm{ppm}) & : 1.000 \text { y } 2.000 \\ \text { Temperatura }\left({ }^{\circ} \mathrm{C}\right) & : 20 \\ \text { Razón acuoso/resina (vol.) } & : 10 / 1 \text { a } 500 / 1 \\ \text { Tiempo (hrs) } & : 24\end{array}$

Al término de cada prueba se analizaba la solución final por hierro utilizando un espectrómetro de absorción atómica.

\subsection{Pruebas cinéticas}

Se realizaron utilizando un matraz de 0,5 1 con un agitador mecánico. La velocidad de agitación se

Tabla I. Análisis químico de las resinas de intercambio iónico empleada

Table I. Chemical analysis of ion exchange resins

\begin{tabular}{lcccccr}
\hline \multicolumn{1}{c}{ Resina } & C & H & N & O & S & P \\
\hline Monophosphonic & 51,96 & 5,87 & $<0,1$ & 24,27 & 4,98 & 12,37 \\
Diphonix 50/100 mallas & 40,31 & 5,32 & 1,52 & 25,14 & 8,28 & 3,66 \\
Diphonix +20 mallas & 42,64 & 5,75 & 1,51 & 32,83 & 9,81 & 4,92 \\
\hline
\end{tabular}


Tabla II. Equivalencia entre volumen de lecho de resina y resina seca

\section{Table II. Equivalence between bed volume and dried resin mass}

\begin{tabular}{lcc}
\hline & $\begin{array}{c}\text { Resina } \\
\text { Monophosphonic }\end{array}$ & $\begin{array}{c}\text { Resina } \\
\text { Diphonix }\end{array}$ \\
\hline Lecho de resina (forma $\left.\mathrm{H}^{+}\right)$ & & \\
$\left(\mathrm{cm}^{3} / \mathrm{g}\right.$ resina seca, $\left.65^{\circ} \mathrm{C}\right)$ & 2,24 & 2,60 \\
\hline
\end{tabular}

mantuvo en un nivel moderado, considerando que aumentos en la agitación no producían cambios en la cinética del proceso. En estas pruebas, una vez que la solución estaba a la temperatura preestablecida se agregaba la resina y se comenzaba a tomar muestras de $1 \mathrm{~cm}^{3}$ de la solución, que se analizaron por hierro. Las variables consideradas en este estudio y sus valores son:

$$
\begin{array}{ll}
\text { Tamaño partícula }(\mu \mathrm{m}): & -417+297 ;-600+417 ; \\
& -850+600 ;-1180+850 \\
\text { Temperatura }\left({ }^{\circ} \mathrm{C}\right) & : 20 ; 35 ; 50 ; 65 \\
\text { Tiempo }(\min ) & : 0 \text { a } 240 \\
\mathrm{Fe}^{3+}(\mathrm{ppm}) & : 1.000
\end{array}
$$

Las pruebas para el efecto del tamaño de partícula solo se realizaron con la resina Monophosphonic, mientras que las demás condiciones se aplicaron para ambas resinas. Adicionalmente a estas pruebas se aplicó la prueba interrumpida para ambas resinas. La solución electrolito utilizada contenía $35 \mathrm{~g} / \mathrm{l}$ de cobre y 180 g/l de ácido sulfúrico.

\section{RESULTADOS EXPERIMENTALES}

Se realizaron pruebas de equilibrio y cinéticas para evaluar ambas resinas. En las pruebas de equilibrio se consideró un tiempo de 24 h en un agitador-vibrador con control de temperatura. Las pruebas cinéticas se realizaron en un sistema con agitación mecánica utilizando un reactor de 0,5 1, tomando muestras de $1 \mathrm{~cm}^{3}$, a diferentes tiempos, para análisis químico. Ambas resinas se acondicionaron con una solución de $160 \mathrm{~g} / \mathrm{l}$ de $\mathrm{H}_{2} \mathrm{SO}_{4}$ antes de utilizarlas

\subsection{Pruebas de equilibrio}

Se realizaron pruebas para determinar las curvas de equilibrio de extracción en las condiciones señaladas en el apartado 3. Los datos experimentales obtenidos se ajustaron a diferentes modelos, encontrándose que la ecuación de Rothmund y Kornfeld (R-K) ${ }^{[6}$ y 7$]$ es la que mejor se ajusta. Para la reacción de intercambio iónico:

$$
\mathrm{Fe}^{3+}+3 \mathrm{RH}=\mathrm{R}_{3} \mathrm{Fe}+3 \mathrm{H}^{+}
$$

la ecuación R-K se expresa como:

$$
\frac{\overline{\mathrm{C}}_{\mathrm{Fe}^{3+}}}{\left(\overline{\mathrm{C}}_{\mathrm{H}^{+}}\right)^{3}}=\mathrm{K}_{\mathrm{H}}^{\mathrm{Fe}}\left(\frac{\mathrm{C}_{\mathrm{Fe}^{3+}}}{\left(\mathrm{C}_{\mathrm{H}^{+}}\right)^{3}}\right)^{\mathrm{p}}
$$

considerando el intercambio de los dos iones, la ecuación se puede expresar como:

$$
\frac{Y_{\mathrm{Fe}}}{\left(1-\mathrm{Y}_{\mathrm{Fe}}\right)^{3}}=\mathrm{K}_{\mathrm{He}}^{\mathrm{Fe}}\left(\frac{\overline{\mathrm{C}}_{0}}{\mathrm{C}_{0}}\right)^{2}\left(\frac{\mathrm{X}_{\mathrm{Fe}}}{\left(1-\mathrm{X}_{\mathrm{Fe}}\right)^{3}}\right)^{p}
$$

$$
\begin{aligned}
& \text { Con: } \\
& Y=\bar{C} / \bar{C}_{0}=\text { fración iónica en la resina } \\
& X=C / C_{0}=\text { fracción iónica en la solución } \\
& \qquad \begin{array}{l}
X_{\mathrm{Fe}}+Y_{H}=1 \\
X_{\mathrm{Fe}}+X_{H}=1
\end{array}
\end{aligned}
$$

Representando gráficamente $\operatorname{Ln}\left(\mathrm{Y}_{\mathrm{Fe}} /\left(1-\mathrm{Y}_{\mathrm{H}}\right)^{3}\right)$ en función de $\operatorname{Ln}\left(X_{\mathrm{Fe}} /\left(1-X_{\mathrm{H}}\right)^{3}\right)$, se obtiene así una línea recta que corta el eje $\mathrm{Y}$ en $\mathrm{Ln}\left(\mathrm{K}_{\mathrm{H}}^{\mathrm{Fe}}\left(\overline{\mathrm{C}}_{0} / \mathrm{C}_{0}\right)^{2}\right)$.

En las figuras 1 y 2 se muestran los resultados experimentales y el ajuste al modelo $\mathrm{R}-\mathrm{K}$ para la resina Monophosphonic y en la figura 3 para la resina Diphonix.

La figura 1 muestra el efecto en la carga de la resina al contactarla con soluciones que contienen $1.000,1.500$ y $2.000 \mathrm{mg} / \mathrm{l} \mathrm{de} \mathrm{Fe}^{3+}$. Se observa que la concentración máxima de hierro en la resina aumenta desde $55 \mathrm{mg} / \mathrm{g}$, al equilibrarla con una solución de $1.000 \mathrm{mg} / \mathrm{l} \mathrm{de} \mathrm{Fe} \mathrm{a} \mathrm{un} \mathrm{valor} \mathrm{por} \mathrm{encima} \mathrm{de} 80 \mathrm{mg} / \mathrm{g}$ de hierro, al equilibrarla con una solución de $2.000 \mathrm{mg} / \mathrm{l}$ a temperatura ambiente. De la misma forma, la figura 2 muestra que la concentración máxima de hierro en la resina Monophosphonic aumenta con la temperatura desde, aproximadamente $55 \mathrm{mg} / \mathrm{g}$ a temperatura ambiente hasta $70 \mathrm{mg} / \mathrm{g}$ a $55^{\circ} \mathrm{C}$, utilizando una solución de $1.000 \mathrm{mg} / \mathrm{l} \mathrm{de} \mathrm{Fe}^{3+}$. En ambas figuras se observa, además, un buen ajuste del modelo $\mathrm{R}-\mathrm{K}$ a los datos experimentales.

En la figura 3 se observa una menor capacidad de carga de la resina Diphonix con respecto a la Monophosphonic. Para una solución con $1.000 \mathrm{mg} / \mathrm{l}$ de $\mathrm{Fe}^{3+}$ se alcanza un contenido máximo de $32 \mathrm{mg} / \mathrm{g}$ de Fe en la resina, valor que está por debajo de los 


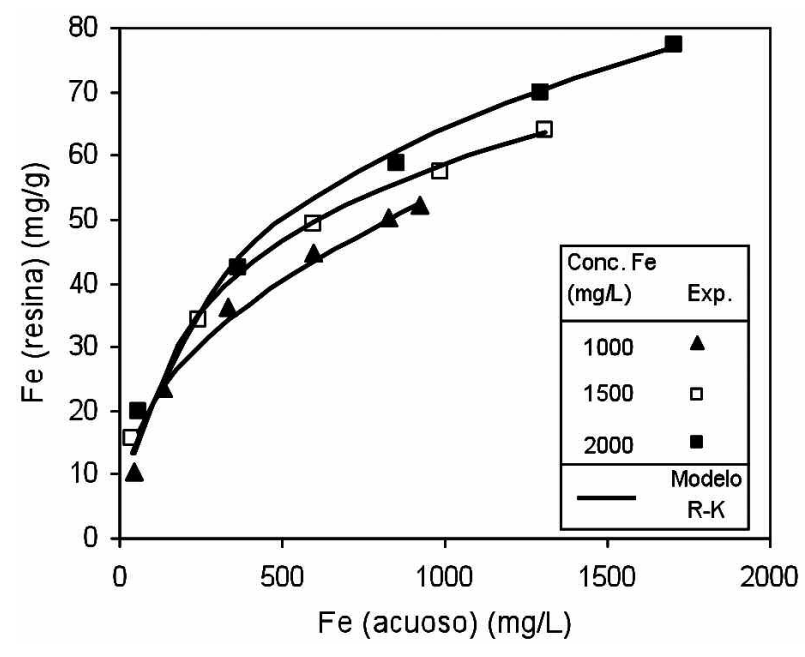

Figura 1. Efecto de la concentración de hierro en la isoterma de carga de la resina Monophosphonic a temperatura ambiente $\left(20^{\circ} \mathrm{C}\right)$.

Figure 1. Iron concentration effect on the loading isotherm of the Monophosphonic resin at room temperature.

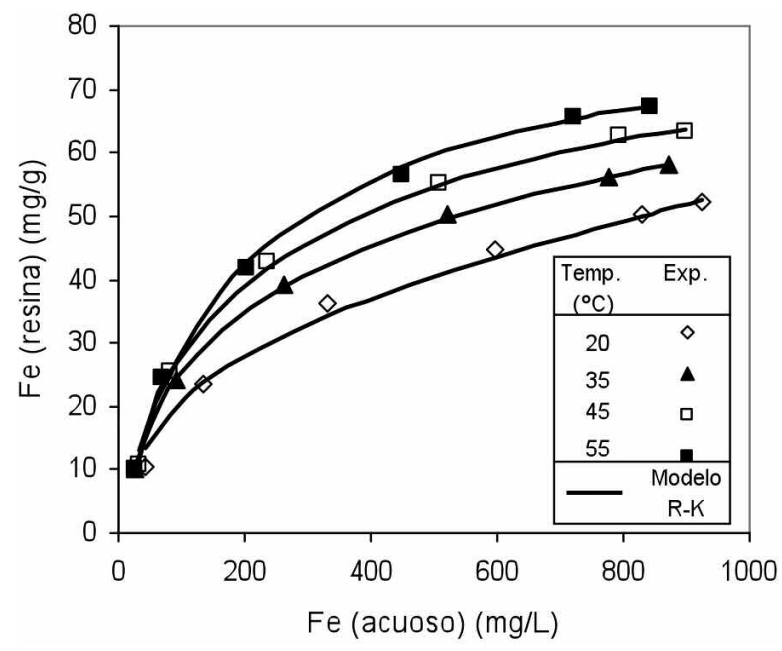

Figura 2. Efecto de la temperatura en la isoterma de carga de la resina Monophosphonic para una solución de $1.000 \mathrm{mg} / \mathrm{l}$ de hierro.

Figure 2. Temperature effect on the loading isotherm of the Monophosphonic resin using a $1000 \mathrm{mg} / \mathrm{l}$ of iron solution.

$55 \mathrm{mg} / \mathrm{g}$ obtenido con la resina Monophosphonic. Esta diferencia se debe al mayor contenido de grupos de ácido fosfónico en la resina Monophosphonic, el cual es el grupo selectivo por el $\mathrm{Fe}^{3+}$.

La ecuación de R-K se ajusta bien a los datos experimentales para ambas resinas, obteniéndose los

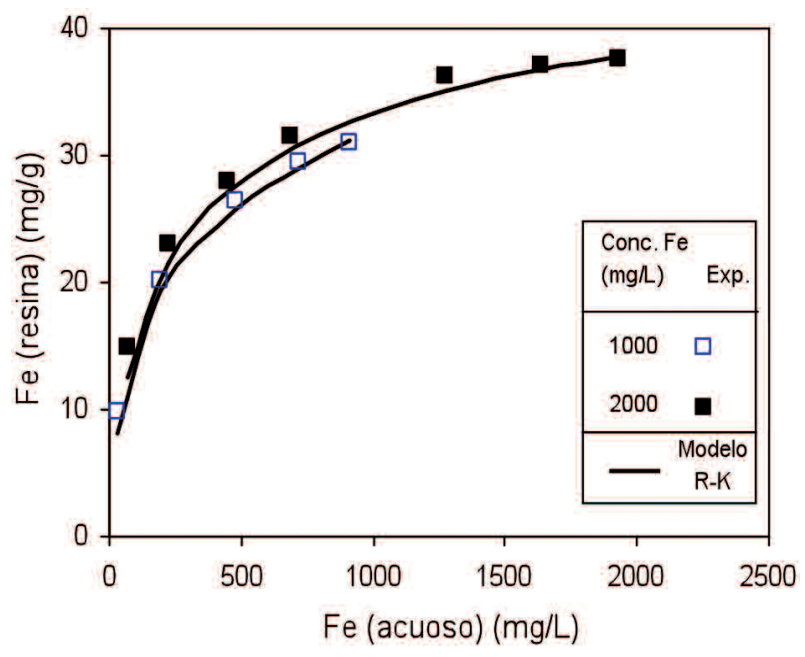

Figura 3. Efecto de la concentración de hierro en la isoterma de carga de la resina Diphonix a temperatura ambiente $\left(20^{\circ} \mathrm{C}\right)$.

Figure 3. Iron concentration effect on the loading isotherm of the Diphonix resin at room temperature.

parámetros del modelo indicados en la tabla III. La constante de equilibrio K para la resina Diphonix es, notoriamente, más alta que para la resina Monophosphonic. El parámetro p es cercano a 1, tanto para la resina Monophosphonic como para la Diphonix, a temperatura ambiente, lo que significa que el valor de $\mathrm{K}$ es equivalente al coeficiente de selectividad. A mayor temperatura con la resina Monophosphonic el parámetro p aumenta, lo cual indica una condición más favorable para la extracción del hierro.

\subsection{Pruebas cinéticas}

En intercambio iónico de resinas está demostrado que la difusión de los iones es el mecanismo que controla la velocidad del proceso $^{[7]}$, habiendo dos posibilidades de control individuales:

- Transporte de los iones en la película de concentración que rodea las partículas de resina.

- Difusión de los iones dentro de la resina.

Las pruebas cinéticas tuvieron por finalidad dilucidar los factores que influyen en la cinética de extracción de hierro, considerando estos dos mecanismos de control. Los datos experimentales obtenidos se ajustaron a diferentes modelos cinéticos, hallándose que con el modelo de difusión de Fick se obtenía el mejor ajuste. Una solución aproximada para la ecuación de Fick es la desarrollada por Vermeulen ${ }^{[7]}$ : 
EVALUACIÓN DE RESINAS DE INTERCAMBIO IÓNICO PARA EL CONTROL DEL HIERRO EN SOLUCIONES DE ELECTRO-OBTENCIÓN DE COBRE EVALUATION OF ION EXCHANGE RESINS FOR IRON CONTROL IN COPPER ELECTRO-WINNING SOLUTIONS

Tabla III. Parámetros de la ecuación de Rothmund y Kornfeld para las isotermas de carga

Table III. The Rothmund and Kornfeld parameters obtained for the loading isotherms

\begin{tabular}{lcccccc}
\hline Resina & $\begin{array}{c}\text { Hierro en } \\
\text { solución } \\
(\mathbf{m g} / \mathbf{l})\end{array}$ & $\begin{array}{c}\text { Temperatura } \\
\mathbf{(} \mathbf{C})\end{array}$ & $\begin{array}{c}\text { Max carga } \\
\text { de hierro } \\
\mathbf{( m g / g )}\end{array}$ & $\mathbf{K}$ & $\mathbf{p}$ & $\mathbf{R}^{\mathbf{2}}$ \\
& 1.000 & 20 & 55 & 3.723 & 0,96 & 0,9891 \\
& 1.500 & 20 & 66 & 13.301 & 1,17 & 0,9948 \\
Monophosphonic & 2.000 & 20 & 90 & 2.996 & 0,68 & 0,9823 \\
& 1.000 & 35 & 60 & 7.483 & 1,22 & 0,9992 \\
& 1.000 & 45 & 65 & 8.565 & 1,33 & 0,9935 \\
Diphonix & 1.000 & 55 & 68 & 9.437 & 1,54 & 0,9979 \\
& 1.000 & 20 & 32 & 40.948 & 1,05 & 0,9964 \\
& 2.000 & 20 & 38,5 & 78.282 & 0,64 & 0,9391 \\
\hline
\end{tabular}

$\mathrm{K}((\mathrm{eq} / \mathrm{L}) /(\mathrm{eq} / \mathrm{g}))^{2}$.

$$
X=\left(1-\exp \left(-\frac{D^{s} \pi^{2} t}{r_{0}^{2}}\right)\right)^{0.5}
$$

O:

$$
\operatorname{Ln}\left(1-X^{2}\right)=-\frac{D^{s} \pi^{2} t}{r_{0}^{2}}
$$

en que, $\mathrm{X}$ es la fracción transformada, $\mathrm{D}$, el coeficiente de difusión, $\mathrm{r}_{0}$, el radio de la partícula de resina y t, el tiempo.

\subsubsection{Efecto del tamaño de partícula}

Se emplearon cuatro tamaños de resina Monophosphonic para determinar su efecto sobre la velocidad de intercambio iónico y, así, ayudar a dilucidar entre un control por transporte en la película que rodea la partícula o difusión en el interior de la partícula de resina.

En la figura 4 se muestran los resultados experimentales y el ajuste a un modelo difusional (modelo de Fick), para control por difusión en el interior de las partículas. Se puede ver que hay un buen ajuste de los datos experimentales al modelo y un significativo efecto del tamaño de partícula en la velocidad de intercambio. El ajuste al modelo, por si solo, no es suficiente para definir el tipo de control, pero indicaría que existiría control por difusión en la partícula.

El efecto del tamaño de partícula en la velocidad del proceso es un criterio frecuentemente empleado para

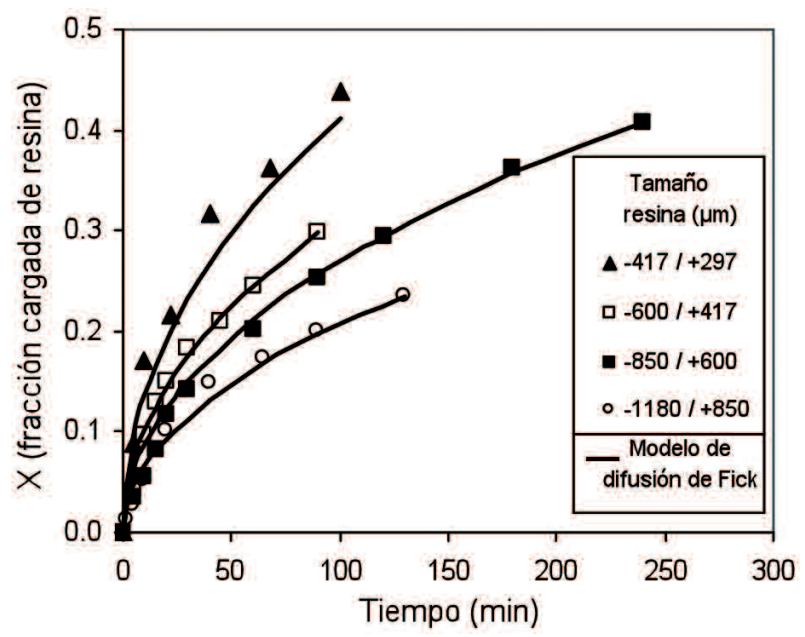

Figura 4. Efecto del tamaño de partícula de resina en la etapa de carga de la resina Monophosphonic a temperatura ambiente (solución. $\left.1 \mathrm{~g} / \mathrm{l} \mathrm{Fe}, 35 \mathrm{~g} / \mathrm{l} \mathrm{Cu}, 180 \mathrm{~g} / \mathrm{l} \mathrm{H}_{2} \mathrm{SO}_{4}\right)$.

Figure 4. Resin particle size effect on the loading stage of the Diphonix resin at room temperature (Loading solution. $1 \mathrm{~g} / \mathrm{l} \mathrm{Fe}, 35 \mathrm{~g} / \mathrm{l} \mathrm{Cu}, 180 \mathrm{~g} / \mathrm{l}$ $\mathrm{H}_{2} \mathrm{SO}_{4}$ ).

discriminar entre los dos tipos de control: para control por transporte en la película de concentración, la velocidad es inversamente proporcional al tamaño de la partícula, mientras que para difusión en el interior de la partícula, la velocidad es inversamente proporcional al cuadrado del tamaño de la partícula ${ }^{[7]}$.

A partir de los datos experimentales se determinaron las velocidades de intercambio empleando las 
tangentes en los puntos experimentales mostrados en la figura 4. En la figura 5, se observa una linealidad casi perfecta al representar gráficamente estas velocidades en función del inverso del cuadrado del tamaño de partícula, es decir, concuerda con el mecanismo de difusión. Sin embargo, este resultado no es categórico, pues al representar gráficamente las velocidades en función del inverso del tamaño de partícula, se obtiene buena linealidad también, pero con un factor de ajuste inferior que en el caso del cuadrado del tamaño.

\subsubsection{Prueba interrumpida}

Esta es una técnica empleada para discriminar entre difusión en el interior de la partícula y en la película que rodea a la partícula ${ }^{[7-9]}$. Para ello, durante la experiencia se retira la resina del reactor y se deja en reposo para luego volver a introducir la resina en la solución y continuar el proceso de intercambio iónico. El tiempo de reposo permite que cualquier gradiente interior de concentración en la resina desaparezca. De esta forma, al retornar la resina al reactor, si el control es por difusión al interior de la partícula, la velocidad de intercambio será mayor que antes de retirarla de la solución. Si el control fuera por

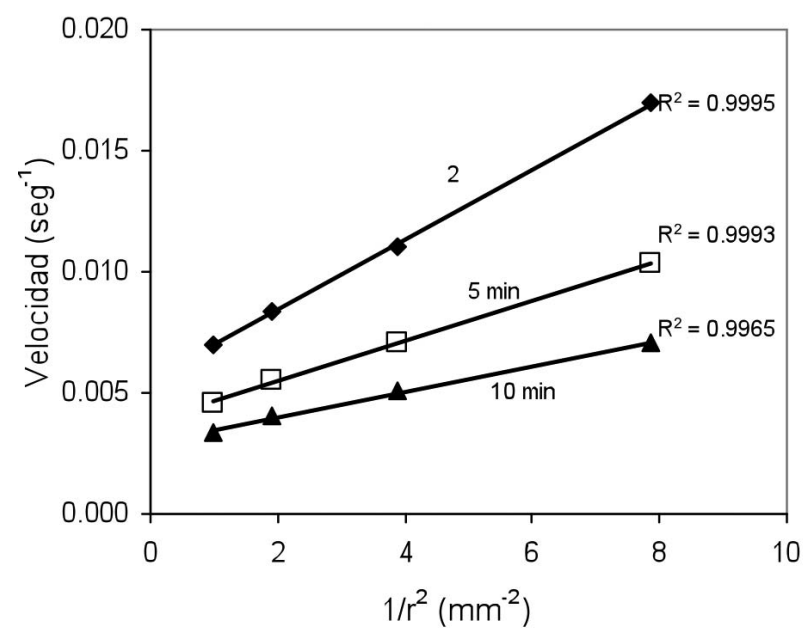

Figura 5. Relación entre la velocidad instantánea de intercambio iónico y el tamaño de partícula para la resina Monophosphonic a temperatura ambiente (solución. $1 \mathrm{~g} / \mathrm{l} \mathrm{Fe}, 35 \mathrm{~g} / \mathrm{l} \mathrm{Cu}, 180 \mathrm{~g} / \mathrm{l}$ $\mathrm{H}_{2} \mathrm{SO}_{4}$ ).

Figure 5. Relationship between instant rate of exchange and the inverse of the square particle size for loading kinetics of monophosphonic resin at room temperature (Solution. $1 \mathrm{~g} / \mathrm{Fe}, 35 \mathrm{~g} / \mathrm{ICu}$, $180 \mathrm{~g} / \mathrm{H} \mathrm{H}_{2} \mathrm{SO}_{4}$ ). transferencia en la película que rodea a la partícula, no existe gradiente de concentración en el interior de la partícula, por lo tanto no habrá variación de la velocidad de intercambio.

En las figuras 6 y 7 se muestran los resultados de estas pruebas para las resinas Monophosphonic y

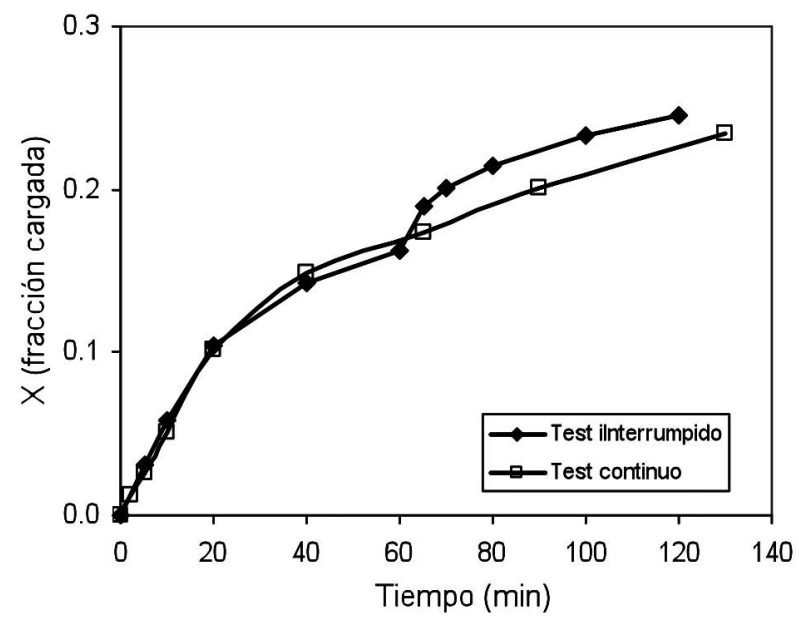

Figura 6. Prueba interrumpida para la cinética de carga de la resina Monophosphonic a temperatura ambiente (solución. $1 \mathrm{~g} / \mathrm{l} \mathrm{Fe}, 35 \mathrm{~g} / \mathrm{l} \mathrm{Cu}, 180$ g/l $\mathrm{H}_{2} \mathrm{SO}_{4}$ ).

Figure 6. Interrupted test for loading kinetics of monophosphonic resin at room temperature (Solution. $1 \mathrm{~g} / \mathrm{l} \mathrm{Fe}, 35 \mathrm{~g} / \mathrm{l} \mathrm{Cu}, 180 \mathrm{~g} / / \mathrm{H}_{2} \mathrm{SO}_{4}$ ).

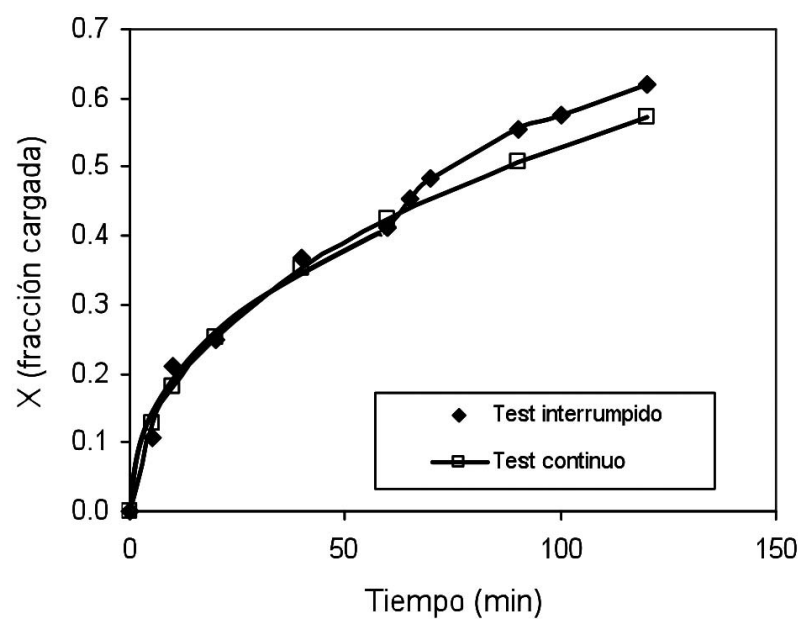

Figura 7. Prueba interrumpida para la cinética de carga de la resina Diphonix a temperatura ambiente (solución. $1 \mathrm{~g} / \mathrm{l} \mathrm{Fe}, 35 \mathrm{~g} / \mathrm{l} \mathrm{Cu}, 180 \mathrm{~g} / \mathrm{l}$ $\mathrm{H}_{2} \mathrm{SO}_{4}$ ).

Figure 7. Interrupted test for loading kinetics of Diphonix resin at room temperature (Solution. 1 $\mathrm{g} / \mathrm{l} \mathrm{Fe}, 35 \mathrm{~g} / \mathrm{l} \mathrm{Cu}, 180 \mathrm{~g} / / \mathrm{H}_{2} \mathrm{SO}_{4}$ ). 
EVALUACIÓN DE RESINAS DE INTERCAMBIO IÓNICO PARA EL CONTROL DEL HIERRO EN SOLUCIONES DE ELECTRO-OBTENCIÓN DE COBRE EVALUATION OF ION EXCHANGE RESINS FOR IRON CONTROL IN COPPER ELECTRO-WINNING SOLUTIONS

Diphonix en las que se observa el cambio de velocidad, lo cual verifica la presencia del control por difusión en el interior de partículas de resina.

\subsubsection{Efecto de la temperatura}

Se evaluó la cinética de extracción de hierro desde una solución con $1 \mathrm{~g} / \mathrm{l}$ de $\mathrm{Fe}$, entre 20 y $65^{\circ} \mathrm{C}$, para ambas resinas. Los resultados se presentan en las figuras 8 y 9. Como se podía esperar, a mayor temperatura más rápida es la cinética de extracción para ambas resinas. Se encontró que la resina Diphonix muestra una cinética más rápida que la Monophosphonic. Por ejemplo, en la figura 8 se observa que, para $60 \mathrm{~min}$, la resina Monophosphonic ha alcanzado entre un $15 \mathrm{y}$ un $35 \%$ de la capacidad de carga en el rango de temperatura ensayado, en tanto que la resina Diphonix (Fig. 9) alcanza entre un 30 y un $50 \%$ de la capacidad de carga para el mismo tiempo de adsorción. Esta diferencia es el reflejo del mayor contenido de ácido sulfónico de la resina Diphonix, el cual se agrega para mejorar la cinética de intercambio iónico.

Como se ve en las figuras 8 y 9 , el modelo difusivo de Fick (Ecs. (4) y (5)) para control por difusión en el interior de la partícula se ajusta bien a todos los resultados experimentales. A partir de la ecuación (5) y representando gráficamente $\operatorname{Ln}\left(1-X^{2}\right)$ en función del tiempo se obtiene una línea recta cuya pendiente permite obtener el valor del coeficiente de difusión. La tabla IV muestra los valores calculados del

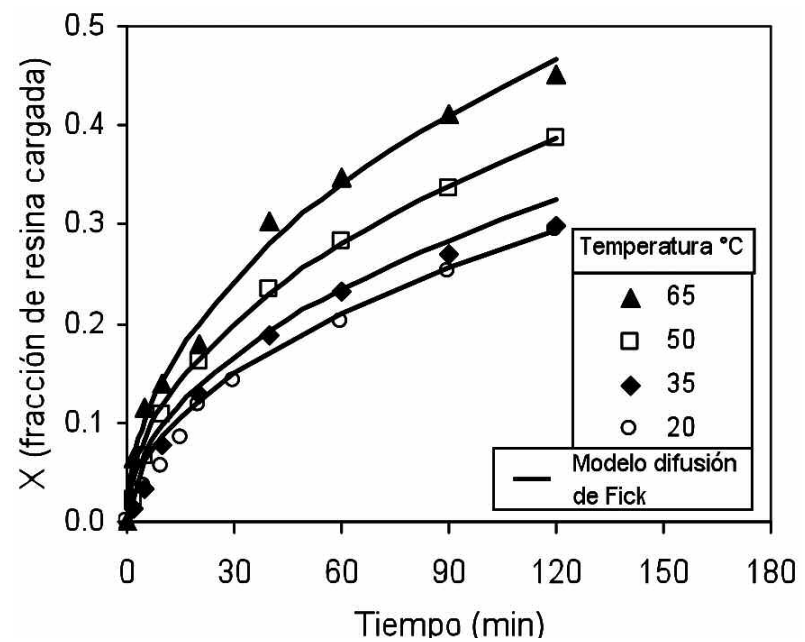

Figura 8. Efecto de la temperatura en la cinética de carga de la resina Monophosphonic (solución. $\left.1 \mathrm{~g} / \mathrm{l} \mathrm{Fe}, 35 \mathrm{~g} / \mathrm{l} \mathrm{Cu}, 180 \mathrm{~g} / \mathrm{H} \mathrm{H}_{2} \mathrm{SO}_{4}\right)$.

Figure 8. Temperature effect on the loading kinetics of monophosphonic resin (Solution. 1 $\mathrm{g} / \mathrm{I} \mathrm{Fe}, 35 \mathrm{~g} / \mathrm{l} \mathrm{Cu}, 180 \mathrm{~g} / \mathrm{H} \mathrm{H}_{2} \mathrm{SO}_{4}$ ).

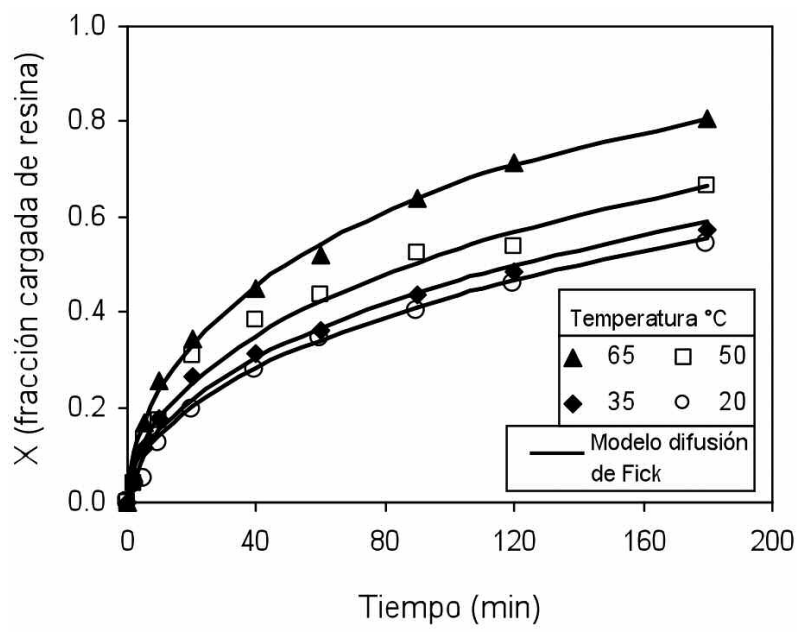

Figura 9. Efecto de la temperatura en la cinética de carga de la resina Diphonix (solución. 1 $\mathrm{g} / \mathrm{l} \mathrm{Fe}, 35 \mathrm{~g} / \mathrm{l} \mathrm{Cu}, 180 \mathrm{~g} / \mathrm{l} \mathrm{H}_{2} \mathrm{SO}_{4}$ ).

Figure 9. Temperature effect on loading kinetics of Diphonix resin (Solution. $1 \mathrm{~g} / \mathrm{l} \mathrm{Fe}, 35 \mathrm{~g} / \mathrm{L} \mathrm{Cu}$, $180 \mathrm{~g} / \mathrm{I} \mathrm{H}_{2} \mathrm{SO}_{4}$ ).

Tabla IV. Coeficiente de difusión para la etapa de carga

Table IV. Difusión coefficient for loading stage

\begin{tabular}{ccc}
\hline & \multicolumn{2}{c}{ Coeficiente de difusión $\left(\mathrm{cm}^{2} / \mathrm{s}\right)$} \\
\cline { 2 - 3 } Temperatura ${ }^{\circ} \mathrm{C}$ & Monophosphonic & Diphonix \\
\hline 20 & $6,74 \times 10^{-13}$ & $1,46 \times 10^{-12}$ \\
35 & $8,30 \times 10^{-13}$ & $2,13 \times 10^{-12}$ \\
50 & $1,21 \times 10^{-12}$ & $2,89 \times 10^{-12}$ \\
65 & $1,81 \times 10^{-12}$ & $4,13 \times 10^{-12}$ \\
\hline
\end{tabular}

coeficiente de difusión en función de la temperatura para ambas resinas. Se puede observar que la resina Diphonix tiene un mayor valor del coeficiente de difusión que la resina Monophosphonic, lo que es coherente con la cinética intercambio más rápida con esta resina. El error en el cálculo del coeficiente de difusión, se puede asociar al valor de $\mathrm{R}^{2}$ obtenido al ajustar los datos experimentales con la ecuación de Fick y que, en el caso de la resina Monophosphonic, varió entre 0,9897 y 0,9990, mientras que para la resina Diphonix el valor de $\mathrm{R}^{2}$ varió de 0,9795 a 0,9949. En ambos casos, se puede afirmar que los valores del coeficiente de difusión calculados representan bien los datos experimentales. 
Empleando los valores calculados del coeficiente de difusión en función de la temperatura se puede verificar si sigue un comportamiento tipo Arrhenius y determinar la energía de activación asociada al proceso de intercambio. El valor de esta energía de activación es otro criterio para discriminar entre los tipos de control que pueden existir en la cinética del proceso de intercambio iónico, los cuales se identifican con valores bajos de energía de activación debido a la poca dependencia de la cinética con respecto a la temperatura y que es característico de los procesos controlados por transferencia de masa.

Representando gráficamente el logaritmo del coeficiente de difusión en función del inverso de la temperatura, como se muestra en la Figura 10 a) y b), se obtiene una línea recta cuya pendiente permite calcular el valor de la energía de activación. El valor obtenido para la energía de activación de ambas resinas se muestra en la tabla V.

Los valores calculados para la energía de activación están en el rango de procesos controlados por difusión en la solución: 3 - $6 \mathrm{kcal} / \mathrm{mol}$ y por debajo del valor para difusión en resinas: $6-10 \mathrm{kcal} / \mathrm{mol}^{[7]}$. La energía de activación en procesos de intercambio iónico es afectada por la naturaleza de los iones y el tamaño de los poros de la resina. Un ión pequeño puede no ser afectado por el efecto pared y moverse libremente a través de la solución que llena los poros de la resina.

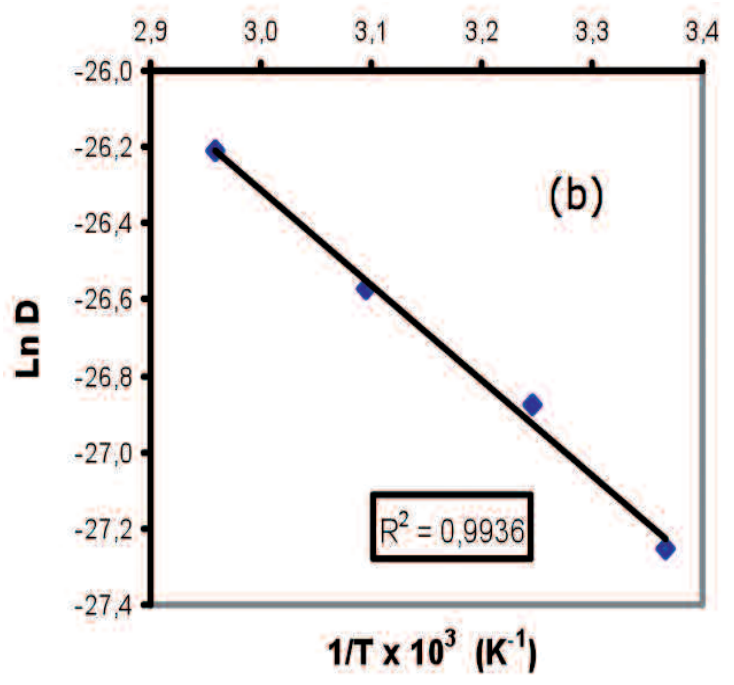

Tabla V. Energía de activación

Table V. Activation energy

\begin{tabular}{ccc}
\hline \multirow{2}{*}{$\begin{array}{c}\text { Rango } \\
\text { temperatura }\end{array}$} & \multicolumn{2}{c}{ Energía de activación (kcal/mol) } \\
\cline { 2 - 3 } & $\begin{array}{c}\text { Resina } \\
\text { Monophosphonic }\end{array}$ & $\begin{array}{c}\text { Resina } \\
\text { Diphonix }\end{array}$ \\
\hline $20-65^{\circ} \mathrm{C}$ & 4,85 & 4,94 \\
\hline
\end{tabular}

\section{CONCLUSIONES}

Las dos resinas evaluadas pueden extraer en forma eficiente el Fe(III) desde soluciones de EW de cobre. Por ejemplo, para una electrolito de electro-obtención de cobre con 1.000 ppm de $\mathrm{Fe}^{3+}$, se alcanza un $95 \%$ de eliminación de hierro.

Las diferencias en el rendimiento de las resinas son reflejo de las diferencias en su composición. La resina Diphonix muestra una cinética más rápida y que es coherente con el mayor contenido de grupos de ácido sulfónico, mientras que la resina Monophosphonic posee mayor capacidad de carga producto del mayor contenido de grupos de ácido fosfónico, selectivos para Fe(III).

Las isotermas de extracción de las dos resinas evaluadas quedan bien representadas por la ecuación empírica de Rothmund y Kornfeld.

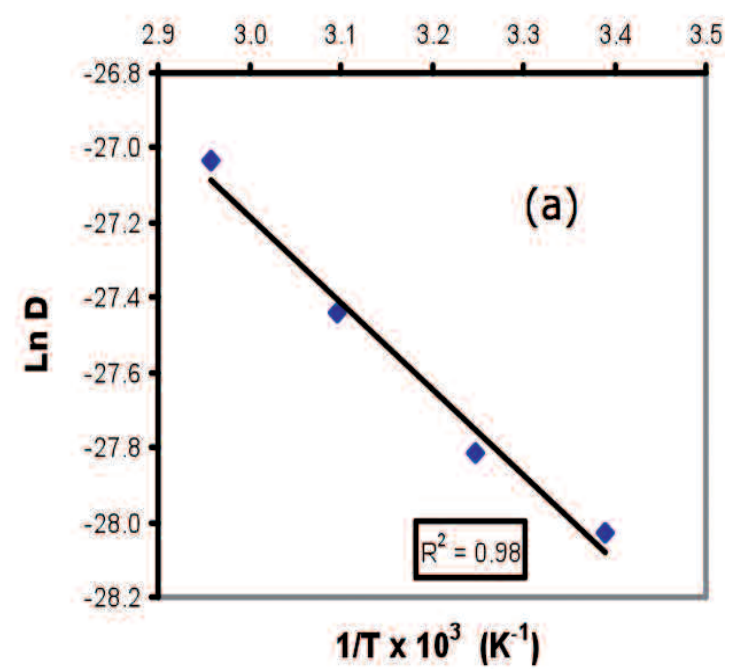

Figura 10. Determinación de la energía de activación, (a) resina Monophosphonic (b) resina Diphonix (solución: $1 \mathrm{~g} / \mathrm{l} \mathrm{Fe}, 35 \mathrm{~g} / \mathrm{l} \mathrm{Cu}, 180 \mathrm{~g} / \mathrm{H}_{2} \mathrm{SO}_{4}$ )

Figure 10. Activation energy determination, (a) monophosphonic resin and (b) Diphonix resin (Loading solution: $1 \mathrm{~g} / \mathrm{l} \mathrm{Fe}, 35 \mathrm{~g} / \mathrm{l} \mathrm{Cu}, 180 \mathrm{~g} / \mathrm{l} \mathrm{H}_{2} \mathrm{SO}_{4}$ ). 
Los resultados obtenidos comprueban que la cinética de extracción de la resina Monophosphonic está controlada por la difusión de los iones $\mathrm{Fe}^{3+}$ en el interior de las partículas de resina. Estos resultados están basados en la siguiente información experimental:

- los datos experimentales se ajustan bien al modelo de difusión de Fick para control por difusión en el interior de las partículas de resina

- existe una relación lineal entre la velocidad de intercambio y el inverso del cuadrado del tamaño de partícula de resina

- la prueba interrumpida muestra un claro aumento en la velocidad de intercambio después del período de reposo de la resina. Esa prueba resulta ser la forma más clara para discriminar entre transporte en la película que rodea a la partícula y difusión en el interior de las partículas.

La temperatura favorece la cinética del proceso de extracción, pero con un débil efecto que se refleja en un bajo valor para la energía de activación e igual a 4,89 kcal/mol para la resina Monophosphonic y $4,94 \mathrm{kcal} / \mathrm{mol}$ para la resina Diphonix. Estos valores confirman un mecanismo de control por transferencia de masa y están cercanos al valor típico para control por difusión en las partículas de resinas de intercambio iónico $(6$ a $10 \mathrm{kcal} / \mathrm{mol})$.

\section{REFERENCIAS}

[1] M.J. Gula, D.B. Dreisinger, J.C. Montijo y S.K. Young, Iron Control and Disposal, Ottawa, Canada, 1996, J.E. Dutrizac y G.B. Harris (Eds.), Canadian Institute of Mining, Metallurgy and Petroleum, Montreal, Canada, pp. 315- 327.

[2] S.S. Xue, M.J. Gula, J.T. Harvey and E.P. Horwitz, SME Annual Meeting, Salt Lake City, EE.UU., 2000.

[3] D.R. Shaw, D.B. Dreisinger, T. Lancaster, G.D. Richmond y M. Tomlinson, JOM (2004) 38-41.

[4] I. Wilkomirsky, F. Parada y R. Parra, Society of the Canadian Institute of Mining, Metallurgy and Petroleum COM 2002.

[5] R. Chiarizia, E.P. Horwitz, S.D. Alexandratos y M.J. Gula, Separ. Sci. Technol. 32 (1997) 1-35.

[6] H.F. Walton, Ion Exhange Equilibria en Ion exchange: theory and application, Academic Press, Primera edición, New York, EEUU, 1949, pp. 3-12.

[7] F. Helfferich, Ion Exchange, McGraw-Hill Book Company, Primera Edición, New York, EE.UU., 1962, 194-308.

[8] T.R. Kressman y J.A. Kitchener, Discuss.s Faraday Soc. 7 (1949) 90.

[9] B. Hering y H. Bliss, A.I.Ch.E. J. 9 (1963) 495503. 